\title{
Sampling fractional Brownian motion in presence of absorption: a Markov Chain method
}

\author{
Alexander K. Hartmann * \\ Institute of Physics, University of Oldenburg, Oldenburg, Germany \\ Satya N. Majumdar and Alberto Rosso \\ Université Paris-Sud, CNRS, LPTMS, UMR 8626, Orsay F-91405, France.
}

(Dated: August 6, 2018)

\begin{abstract}
We study fractional Brownian motion (fBm) characterized by the Hurst exponent $H$. Using a Monte Carlo sampling technique, we are able to numerically generate $\mathrm{fBm}$ processes with an absorbing boundary at the origin at discrete times for a large number of $10^{7}$ time steps even for small values like $H=1 / 4$. The results are compatible with previous analytical results that the distribution of (rescaled) endpoints $y$ follow a power law $P_{+}(y) \sim y^{\phi}$ with $\phi=(1-H) / H$, even for small values of $H$. Furthermore, for the case $H=0.5$ we also study analytically the finite-length corrections to the first order, namely a plateau of $P_{+}(y)$ for $y \rightarrow 0$ which decreases with increasing process length. These corrections are compatible with the numerical results.

PACS numbers:

Keywords: Brownian motion, Hurst exponent, numerical simulations
\end{abstract}

\section{INTRODUCTION}

The Brownian motion plays a key role in modern theoretical physics, as it explains many effects observed in physical systems. It is currently used in various fields of science to understand, for instance, the trend of financial markets, the dynamics of complex molecules within the cell or the animals food-searching strategies. In order to describe the fluctuations in these systems, it is often necessary to go beyond the Brownian motion and consider random walkers whose mean square displacement grows over time in a nonlinear way. The term used to refer to this situation is anomalous diffusion, in particular sub diffusion if the mean square displacement grows less than linearly, super diffusion if it is faster.

In practice, anomalous diffusion occurs whenever the process $x(\tau)$ is self affine (at least at large time) with a characteristic value of the so-called Hurst exponent $H \neq 1 / 2$, so that the displacement grows with time as $\tau^{H}$. A remarkable example of process displaying anomalous diffusion is the fractional Brownian motion (fBm), originally introduced by Mandelbrot [1]. This process is self-affine Gaussian process with $0<H<1$. A Gaussian process is completely defined by its autocorrelation function, which for $\mathrm{fBm}$ writes as

$$
\left\langle x(\tau) x\left(\tau^{\prime}\right)\right\rangle=\tau^{2 H}+\left(\tau^{\prime}\right)^{2 H}-\left|\tau-\tau^{\prime}\right|^{2 H},
$$

where $x(0)=0$ and the brackets $\langle\ldots\rangle$ refer to an ensemble average over the realizations of the Gaussian processes. The strength of the correlation is described by the Hurst exponent. Note that Eq. (1) implies $\left\langle\left[x\left(\tau_{1}\right)-x\left(\tau_{2}\right)\right]^{2}\right\rangle=2\left|\tau_{1}-\tau_{2}\right|^{2 H}$. This means, $H=1 / 2$

*Electronic address: a.hartmann@uni-oldenburg.de corresponds to the Brownian motion (standard diffusion), $H>1 / 2$ to super-diffusive paths, while $H<1 / 2$ correspond to sub-diffusive paths.

Recently these random walks have found to be relevant for many physical applications. Among them we mention the fluctuations of a tagged monomer of an equilibrated Rouse chain [2, 3] or of a tagged particle in the one dimensional system [4, 5]. In both cases the motion of the tagged object can be modeled as a fractional Brownian motion with $H=1 / 4$. Other physical processes such as the mechanical unzipping of DNA [6], the translocation of polymers through nanopores [2, 7 9], and subdiffusion of macromolecules inside cells and membranes [10 12] can be well described by fBm diffusion.

Despite the large number of cases where $\mathrm{fBm}$ is observed, very little is known about the properties of this process when is confined in a domain of the space, which is the case for many of the above mentioned applications. A concrete example is given by the process of polymer translocation. In this case it has been shown [2, 7] that the fraction of the polymer penetrated inside the nanopore fluctuates with time as a $\mathrm{fBm}$ walker confined inside the interval 0 (which corresponds to the translocation failure) and 1 (which corresponds to completed translocation).

In presence of boundaries the translational invariance is lost and analytical representations like the fractional Langevin equation are of little help [13]. In these situations, numerical simulations remain the option to answer many concrete questions arising from biology and physics 14-16]. Recently, methods where developed to stdy fBm for a system with a potential [17] and for $\mathrm{fBm}$ in confined geometries [18]. Here we present a new generic numerical method which we use here to study these processes in presence of boundaries. We will study in detail the case where there is an absorbing wall in $x=0$ : we thus consider only the paths that remain positive up to 
the final time $\tau$. Recently, analytical predictions [7, 19] were obtained for the distribution $P_{+}(y)$ of rescaled motion endpoints $y \sim x /\left(\tau^{H}\right)$ at end time $\tau$. A possible numerical strategy consists in the direct sampling of $L$ step fBm paths $x_{0}, x_{1}, \ldots, x_{L}$ at discrete times, starting at $x_{0}=0$. This strategy is demanding, in particular for $H<1 / 2$, since in presence of an absorbing boundary the success probability of generating a non-absorbed trajectory is very small. Hence, such simulations were restricted to a small number $L$ of discrete steps. Here, using a Markov Chain approach, we were able to generate long $\mathrm{fBm}$ processes up to $L=10^{7}$ discrete steps for values such as $H=1 / 4,2 / 5,1 / 2,2 / 3$ and $3 / 4$.

The outline of the remainer of the paper is as follows: Next, we present our numerical approach and then we present our numerical results: First - in order to verify that our approach is working- we consider the case of Brownian motion $(H=1 / 2)$, where analytical results for the finite-length corrections are available. Furthermore we study the cases $H=1 / 4, H=2 / 5$ and $H=2 / 3$, $H=3 / 4$ as examples for the two regions $H<1 / 2$ and $H>1 / 2$. In all cases the results are compatible with previous analyitcial predictions. Finally, we summarize our results.

\section{NUMERICAL METHODS}

To generate $\mathrm{fBm}$ processes on a computer, we study discrete-time random walks with suitable correlations. It is useful to introduce the increments of the random walk, namely $\Delta x_{l}=x_{l+1}-x_{l}$. For Gaussian processes the increments are Gaussian variables defined by their autocorrelation function. Using Eq. (11) we can compute the autocorrelation function of the Gaussian increments:

$$
\begin{aligned}
C_{l+m, l} & \equiv\left\langle\Delta x_{l+m} \Delta x_{l}\right\rangle \\
& =|m+1|^{2 H}-2|m|^{2 H}+|m-1|^{2 H} \equiv C(m)
\end{aligned}
$$

We note that this function is independent of the initial time $l$. Matrices having this property are called Toeplitz matrices. Moreover thus the increments are identical Gaussian numbers with variance $\sigma^{2}=2$ displaying power law correlations. Taking the limit $m \rightarrow \infty$ it is easy to extract the power law decay of these correlations. For super diffusive $\mathrm{fBm}(H>1 / 2), C(m)$ is positively correlated with a decay as $m^{-2(1-H)}$. Positive correlation means that there is a high probability to observe a long sequence of increments of same sign. For sub diffusive $\mathrm{fBm}(H<1 / 2), C(m)$ is negatively correlated and decay as $-m^{-2(1-H)}$. Negative correlation means that there is a high probability to observe a long sequence of increments of oscillating sign.

The direct generation of $L$ steps with increment correlation Eq.(2) is straightforward, in principle. The starting point is a vector $\xi=\left(\xi_{0}, \xi_{1}, \ldots, \xi_{L-1}\right)$ of $L$ independent and identically distributed (iid) Gaussian (mean zero, variance one) numbers $\sim G(0,1)$. For the uncorre- lated case $(H=1 / 2)$ one could directly use the random numbers, multiplied by $\sqrt{2}$ to obtain the right $C_{l, l}$, as increments of the $\mathrm{fBm}$ processes, i.e., $x^{\text {uncorr }}(L)=$ $\sum_{l=0}^{L-1} \sqrt{2} \xi_{l}$.

For the case $H \neq 1 / 2$, since $C$ as a correlation matrix is positive semi-definite, there exist a matrix $A$ such that $C=A^{2}$. Thus, one could use $\Delta x=A \xi$ to obtain a random vector with the desired property Eq. (2). Nevertheless, this is too time consuming, since it requires diagonalizing a $L \times L C$ matrix once ( $\sim L^{3}$ operations) and, for each process, the multiplication with the $L \times L$ matrix, $A$ ( $\sim L^{2}$ operations) 36]. This is not feasible, in practice, given the sizes $L=10^{7}$ we study here.

Instead, we used the circulant embedding method for fast generation of Gaussian field proposed in 20, 21]. This method allows to generate random increments which are approximately correlated according to Eq.(2) by generating a periodic increment sequence of period $L^{\prime}$, with $L^{\prime} \geq 2 L$. The correlation of this periodic sequence are encoded in a covariance matrix $\mathcal{C}_{l, l+m}=\mathcal{C}(m)$ of size $L^{\prime} \times L^{\prime}$ built using the original covariance $C$ and defined as:

$$
\begin{gathered}
\mathcal{C}(m)=C(m) \text { for } m=0, \ldots, L^{\prime} / 2-1 \\
\mathcal{C}(m)=C\left(L^{\prime}-m\right) \quad \text { for } m=L^{\prime} / 2, L^{\prime}-1
\end{gathered}
$$

Toeplitz matrices displaying this periodicity are called circulant matrices. For the actual analysis of the numerical simulations we consider only the first $L$ steps $\Delta x_{1}, \Delta x_{2}, \ldots, \Delta x_{L}$. If $L$ is large, the correlation between the first and the last increment is small and the periodicity has no large influence. The advantage of this approach is that the periodicity of the matrix $\mathcal{C}$ allows the application of Fast Fourier Transformation (FFT) to generate the fBms [22].

The FFT is performed in $\sim L^{\prime} \log \left(L^{\prime}\right)$ operations, technically we use the GNU Scientific Library (GSL) 23]. Let be $\hat{c}_{k}$ the FFT of $\mathcal{C}(m)$ from Eq. (3), i.e., $\hat{c}_{k}=\sum_{m=0}^{L^{\prime}-1} \mathcal{C}(m) e^{-2 \pi i \frac{k}{L^{\prime}} m}$. Since $\mathcal{C}(m)$ is symmetric and positive, the coefficients $\hat{c}_{k}$ are real positive numbers. The generation of the correlated random numbers works as follows.

(i) the starting point are $L^{\prime}$ independent and identically distributed (iid) Gaussian numbers (with zero mean and variance one).

$$
\xi=\left(\xi_{0}, \xi_{1}, \ldots, \ldots, \xi_{L-1}\right) .
$$

(ii) we define

$$
\hat{\delta}_{k}=\sqrt{L^{\prime} \hat{c}_{k}} \xi_{k} \quad\left(k=0, \ldots, L^{\prime}-1\right)
$$

which are real numbers as well and where the factor $\sqrt{L^{\prime}}$ takes into account the correct normalization.

(iii) The vector of the increments is obtained after back transforming the vectors of $\hat{\delta}_{k}$ :

$$
\delta_{l}=\frac{1}{L^{\prime}} \sum_{k=0}^{L^{\prime}-1} \hat{\delta}_{k} e^{2 \pi i \frac{l}{L^{\prime}} k},
$$


and taking real and imaginary part:

$$
\Delta x_{l}=\operatorname{Re}\left\{\delta_{l}\right\}+\operatorname{Im}\left\{\delta_{l}\right\}
$$

with $l=1,2, \ldots, L^{\prime}$.

It is easy to check that these three steps lead to the desired correlation. Using $\xi_{k}=\xi_{k}^{*}$ and $\left\langle\xi_{k} \xi_{k^{\prime}}\right\rangle=\delta_{k, k^{\prime}}$ one arrives at $\left\langle\delta x_{l}^{(*)} \delta x_{j}^{(*)}\right\rangle=C( \pm i \pm j)$ where the first and second signs are + for the case of having $\delta x_{l}$ and $\delta x_{j}$ on the left, respectively, and - for the conjugate complex, respectively Thus, using $\operatorname{Re}(z)=\left(z+z^{*}\right) / 2$, $\operatorname{Im}(z)=\left(z-z^{*}\right) / 2 i$, and $C(m)=C(-m)$ one obtains finally, as desired

$$
\begin{aligned}
&\left\langle\Delta x_{l+m} \Delta x_{l}\right\rangle= \\
& \frac{1}{4}\left\langle( \delta _ { l + m } + \delta _ { l + m } ^ { * } - i \delta _ { l + m } + i \delta _ { l + m } ^ { * } ) \left(\delta_{l}+\delta_{l}^{*}-\right.\right.\left.\left.i \delta_{l}+i \delta_{l}^{*}\right)\right\rangle \\
&=C(m) .
\end{aligned}
$$

A numerical test of the method is shown in Fig. 1, which proves that indeed the generated ranomd numbers follow Eq. (2).

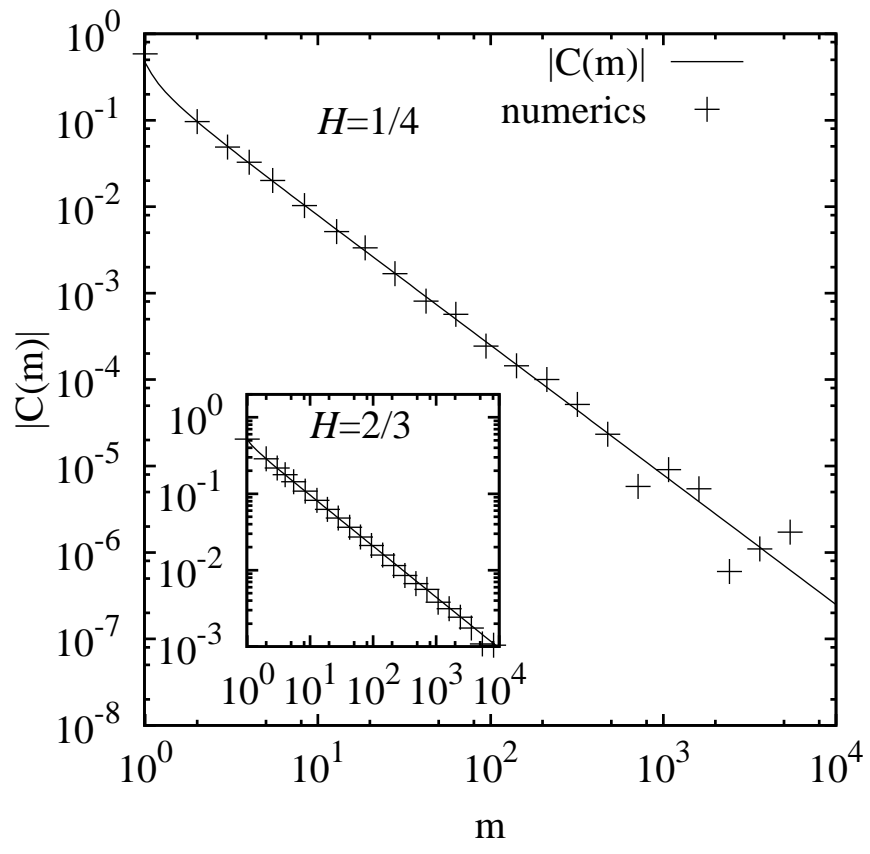

FIG. 1: Correlation between increments. Shown are the wanted function $C(m)$ and the numerical data for $L=10^{4}$. The main plot is for $H=1 / 4$, which the inset displays $H=2 / 3$.

For a direct simulation of $\mathrm{fBm}$ processes, one generates a vector of $L^{\prime}$ real random numbers, constructs the vector of complex numbers, $\xi$, uses the transformation Eqs. (4),(6) to obtain the correlated increments $\Delta x_{l}$ and finally

$$
x \equiv x(L)=\sum_{l=1}^{L} \Delta x_{l}
$$

Nevertheless, since we use an absorbing boundary at $x=0$, most of the time at least one of the intermediate steps will visit the negative half axis, i.e. $\sum_{l=1}^{\ell} \Delta x_{l}<0$ (for some $\ell \leq L$ ), and the obtained value $x(L)$ does not contribute to the distribution $P_{+}(y)$. The probability of being not absorbed, i.e., the persistence (or survival probability), behaves like

$$
S\left(x_{0}=0, L\right) \sim L^{-\theta}
$$

with $\theta$ being the persistence exponent known to be $\theta=$ $1-H[3,24,25]$. Hence, for the case $L=10^{7}$ and $H=1 / 4$, which we study here, we obtain $P_{0}(L) \approx 10^{-5}$. This means, a direct simulation is not feasible. The direct simulation approach has been used in the past [19] only for values of $H \geq 1 / 2$, which is simpler than $H<1 / 2$. Nevertheless, due to the mentioned limitations, even for the simpler case only sizes of $L=20000$ could be studied, in contrast to $L=10^{7}$, which is studied here for values $H \geq 1 / 2$ as well as for $H<1 / 2$. Note that an alternative is to directly simulate a physical process which exibits the nature of fractional Brownian motion, like a suitable polymer model. Nevertheless, this includes many more physical details as a raw $\mathrm{fBm}$, hence only even smaller system can be studied, like in a recent study [26], where polymers of length $N=257$ monomers could be treated.

To circumvent this problem, we performed a Markovchain Monte Carlo simulation with the configuration space being the set of all feasible random vectors $\xi$. Feasible means that the resulting $\mathrm{fBm}$ process (after FFT to generate the correlation of the increments $\Delta x$ ) is not absorbed. The simulation must be initialized with an allowed configuration, namely we start from a random vector $\xi^{(0)}$ and a corresponding correlated increment $\Delta x^{(0)}$ such that the resulting process is not absorbed. In practice, for $H \leq 1 / 2$, we facilitate the generation of a feasible initial configuration by sampling from a shifted Gaussian $G(\bar{\xi}, 1)(\bar{\xi}>0)$ and repeat the search for an initial configurations until one feasible increment vector is found. This initial configuration is clearly biased, but does not have influence on the final result since only after some sufficient equilibration time we start to sample the observables.

Each Monte Carlo step $\xi^{(t)} \rightarrow \xi^{(t+1)}$ consists of changing a fraction $p$ of randomly chosen entries of the configuration $\xi^{(t)}$, the new entries being iid $\mathrm{G}(0,1)$, resulting in a trial configuration $\xi^{\text {trial }}$. Then, again after using FFT to introduce the correlation, we obtain $\Delta x^{\text {trial }}$ : if the resulting $\operatorname{fBm}\left\{\sum_{l=1}^{\hat{L}} \tilde{\Delta} x_{l}^{\text {trial }}\right\}(\hat{L}=1, \ldots, L)$ is absorbed, the trial configuration is rejected, i.e., $\xi^{(t+1)}=\xi^{(t)}$. If the resulting $\mathrm{fBm}$ is allowed, the trial configuration is feasible, hence it is accepted, i.e., $\xi^{(t+1)}=\xi^{\text {trial }}$. This approach satisfies detailed balance, hence converges to the correct distribution: The distribution of the configurations is given by a product of Gaussians over the space of feasible configurations, i.e.,

$$
P(\xi)=\prod_{i=1}^{L^{\prime}}\left(\frac{1}{\sqrt{2 \pi}} \exp \left(-\xi_{i}^{2} / 2\right)\right) I_{\xi}
$$


where the indicator function $I_{\xi}$ is 1 if $\xi$ is feasible, i.e., the resulting $\mathrm{fBm}$ is not absorbed, and 0 else. Hence, if a certain fraction $p$ of the entries of $\xi$ is replaced to yield $\xi^{\prime}$, the resulting change of weight is given by

$$
w\left(\xi \rightarrow \xi^{\prime}\right)=\prod_{j}^{\prime}\left(\frac{1}{\sqrt{2 \pi}} \exp \left(-\xi_{j}^{\prime 2} / 2\right)\right) I_{\xi^{\prime}}
$$

where the product runs over the changed entries. This change of weight is symmetric to the exchange $\xi \leftrightarrow \xi^{\prime}$, hence detailed balance is fulfilled: $P(\xi) w\left(\xi \rightarrow \xi^{\prime}\right)=$ $P\left(\xi^{\prime}\right) w\left(\xi^{\prime} \rightarrow \xi\right)$.

The Markov chain in the configuration space is reflected by the sequence of the endpoints $x^{(0)}(L), x^{(1)}(L), \ldots$ of our Monte Carlo simulation. Here we studied the statistics of the rescaled variable $y=x(L) /\left(\sigma L^{H}\right)$ Since we are interested in the behavior of $P_{+}(y)$ near $y=0$, we also used a bias $b(y)=y^{-a}$ $(a>0)$, by imposing an additional Metropolis criterion [27 29] and accepting a feasible configuration with the probability $p_{\text {accept }}=\min \left\{1, b\left(y^{\text {trial }}\right) / b\left(y^{(t)}\right)\right\}$. This drives the simulation into the range of interest. We adjusted the fraction $p$ of changed entries such that the total acceptance probability of an MC step is near 0.5. Hence, for each value $H$ of the Hurst exponent and each length $L$, we had to find a suitable value $p=p(H, L)$ empirically.

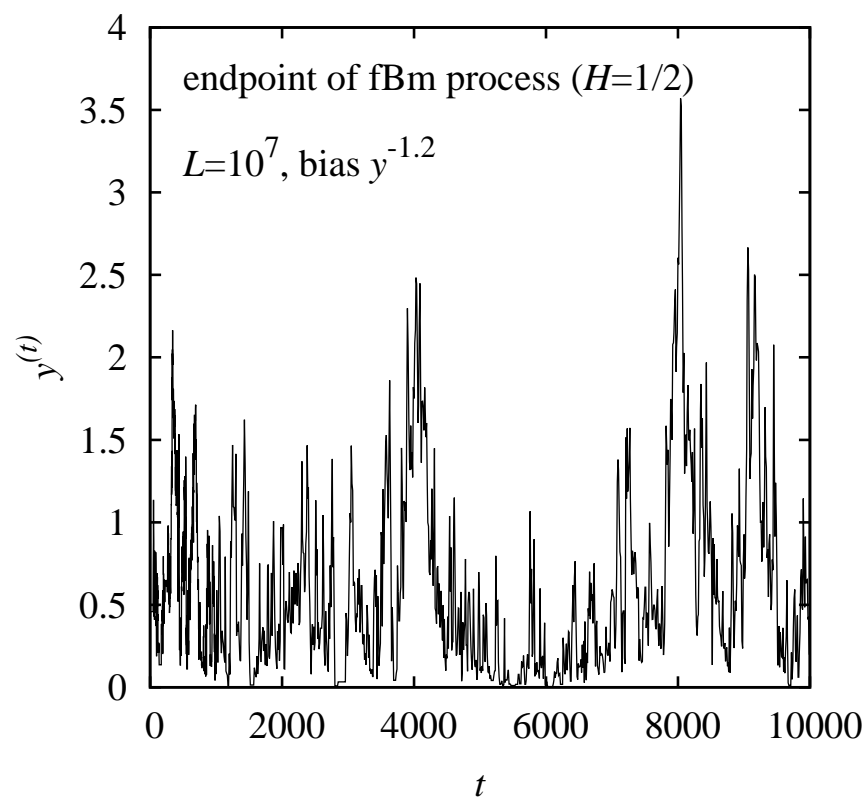

FIG. 2: Sample trajectory of a Monte Carlo simulation: Endpoint $y^{(t)}=x(L)^{(t)} / L^{H}$ of a non-absorbed fBm $(H=1 / 2)$ of length $L=10^{7}$ as a function of the MC time $t$, for the initial phase of the MC simulation up to $t=10^{4}$. A bias $\sim y^{-1.2}$ is used to increase the statistics near $y=0$.

In Fig. 2 a sample trajectory in the space of (rescaled) endpoints is shown for the case $H=1 / 2$ and $L=10^{7}$. Via a bias $b(y) \sim y^{-1.2}$ the simulation is concentrated near $y \approx 0$.

\begin{tabular}{l||c|c|c|c|c}
$L$ & $H=1 / 4$ & $H=2 / 5$ & $H=1 / 2$ & $H=2 / 3$ & $H=3 / 4$ \\
\hline $10^{3}$ & 0.010 & 0.060 & 0.030 & 0.40 & 0.50 \\
$10^{4}$ & 0.020 & 0.020 & 0.020 & 0.40 & 0.50 \\
$10^{5}$ & 0.020 & 0.030 & 0.020 & 0.20 & - \\
$10^{6}$ & 0.010 & 0.020 & 0.020 & 0.10 & - \\
$10^{7}$ & 0.015 & 0.020 & 0.015 & 0.05 & 0.05
\end{tabular}

TABLE I: Value of the Monte Carlo parameter $p$ for different lengths of the fBms and different values of the Hurst exponent $H$.

Concerning equilibration of our Monte Carlo Simulation, we found that typically, for the longest fBm processes, after 1000 sweeps we do not find any sign of the initial configuration. After disregarding this initial bunch of Monte Carlo sweeps, we measured histograms [30] of the rescaled endpoints of the processes. In case a bias is applied, the histograms have to be multiplied by $b^{-1}=y^{a}$ and normalized to get the final distributions $P_{+}(y)$.

Note that the approach use here is rather general: During the Monte Carlo simulation a vector of variables is changed. This vector is evaluated and within a Metropolis criterion is is determined whether the changed vector is accepted. This is like in any Markov chain Monte Carlo simulation, e.g., a single-spin flip simulation of the Ising model. The only difference is that the step transforming the configuration vector into a Metropolis cirtierion is rather involved here since it includes creating a correlation between the vector entries, turning them into random walks, checking for absorption and including a bias keeping the random walks close to the origing. For the Ising system the same step would be just the calculation of an energy. Nevertheless, this approach allows to treat non-equilibrium non-stationary processes, like fractional Brownian motion, within the same framework as conventional equilibrium statistical mechanics systems. Hence, the approach should be applicable to a wide range of problems.

\section{RESULTS}

We have performed simulations to generate $\mathrm{fBms}$ for values of the Hurst exponent $H=1 / 4, H=2 / 5$, $H=1 / 2, H=2 / 3$ and $H=3 / 4$ of lengths $L=10^{3}$, $10^{4}, 10^{5}, 10^{6}$ and $10^{7}$, respectively (for $H=3 / 4$ we did not consider $L=10^{5}$ and $L=10^{6}$ since this was not necessary). For the rescaling, we used $a=2(H=1 / 4)$, $a=1.5(H=2 / 5), a=1.2(H=1 / 2), a=0.5$ $(H=2 / 3)$, and $a=0.4(H=3 / 4)$, respectively. For each case, we determined the parameter $p$, such that the acceptance probability is (very roughly) about 0.5 . The values we used are shown in table $\llbracket$ For each case the MC simulation was performed for long runs, up to $t=3 \times 10^{8}$ for the longest walks of length $L=10^{7}$.

Note that for $H=1 / 4, H=2 / 5$, and $H=1 / 2$, we have restricted the simulations to $\mathrm{fBm}$ processes with $y>$ 
0.0001 to prevent the simulation being caught near $y=0$ due to a very small acceptance ration via the rescaling factor in that region.

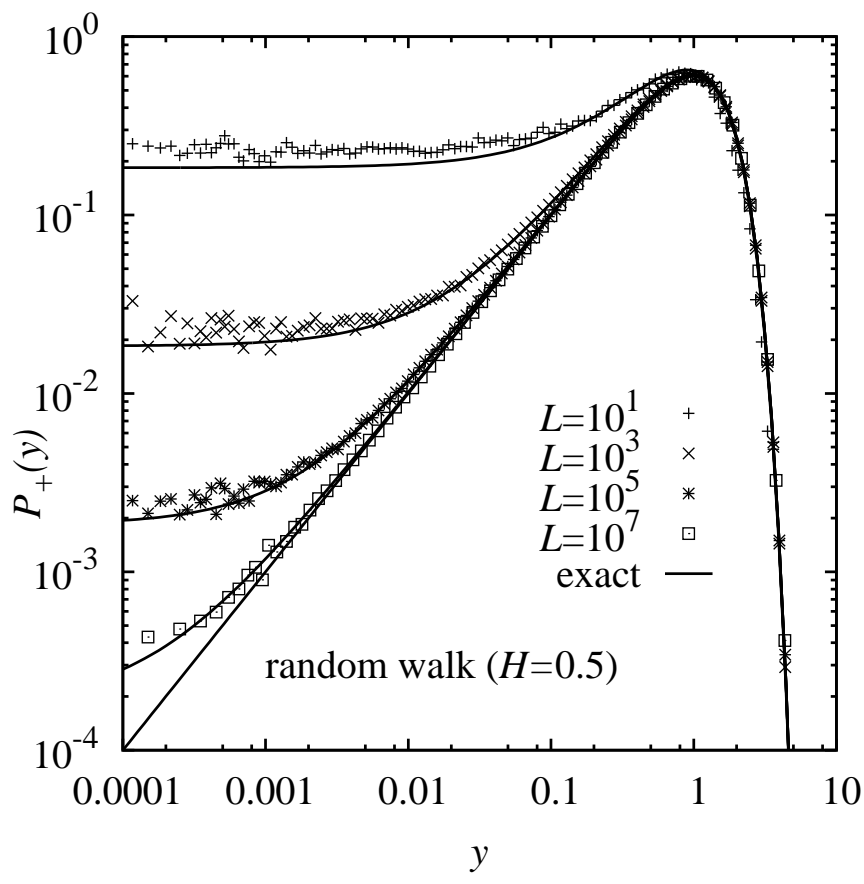

FIG. 3: Distribution $P_{+}(y)$ of rescaled endpoints $y=x / L^{H}$ for non-absorbed fBms (Hurst exponent $H=1 / 2$ )

First, to verify our method, we studied the case of standard random walks, $H=1 / 2$. For this case it is possible to know the first corrections to the continuum limit behavior (see Appendix):

$$
P_{+}(y, L)=f_{0}(y)-\frac{c}{\sqrt{L}} f_{1}(y)+\ldots
$$

where $L$ is the number of increments, the constant $c$ depends on the increments distribution of the random walk. For Gaussian numbers (zero mean, unit variance) we have $c=\zeta(1 / 2) / \sqrt{2 \pi} \sim-0.582597 \ldots$ and the scaling functions are:

$$
\begin{aligned}
& f_{0}(y)=y e^{-y^{2} / 2} \\
& f_{1}(y)=\left(1-\frac{2 y}{\pi}\right) e^{-y^{2} / 2}
\end{aligned}
$$

The rescaled distributions $P_{+}(y)$ of the endpoints are shown, together with the predictions of Eq.(9) valid for large $L$, in Fig. 3. One is able to see strong finite-size effects for small values $y \rightarrow 0$, where a plateau is visible. For increasing length $L$, the plateau decreases as $c / \sqrt{L}$ and the data approaches better and better the continuum limit scaling function $f_{0}$.

We conclude that for a generic $\mathrm{fBm}$ for which first corrections to the continuum limit behavior is not known, the plateau should also vanish when the size of the system is large and for very long walks $\left(L=10^{7}\right)$ the continuum

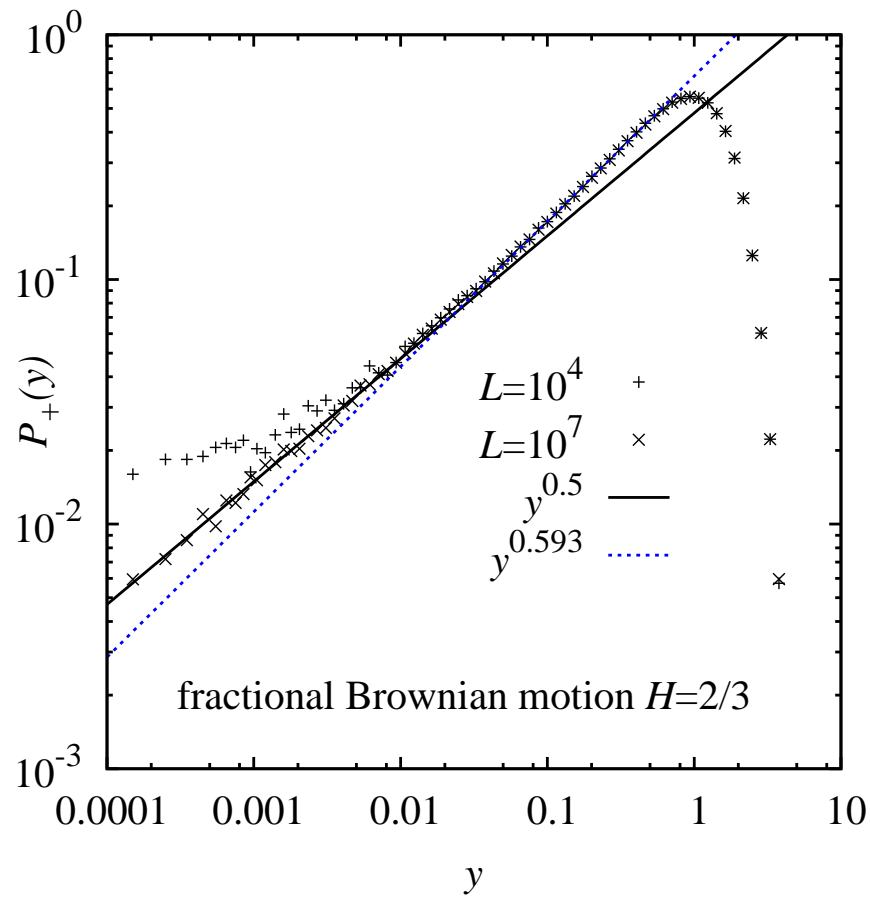

FIG. 4: (color online) Distribution $P_{+}(y)$ of rescaled endpoints $y=x / L^{H}$ for non-absorbed fBms (Hurst exponent $H=2 / 3)$

limit behavior is displayed over several order of magnitudes.

Based on scaling arguments it has been conjectured [7] that, in the continuum limit $P_{+}(y)$ vanishes as $y^{\phi}$ with $\phi=(1-H) / H=\theta / H$ for $y \rightarrow 0, \theta$ being the persistence defined via Eq. (8). This conjecture was confirmed by an epsilon expansion around the Brownian solution obtained thanks to a field theory calculation [19]. The numerical check of the conjecture for values of $H$ far from $1 / 2$ remains very challenging. We first consider the discrete random walk with $H=2 / 3$. In this case, since the persistence is decreasing not very fast $(\theta=1 / 3)$, numerical results were obtained for moderate lengths $L=2 \times 10^{4}$ by direct simulations [19], which were compatible with the analytics. Here, we were able to study this case again. Our results, up to a length of $L=10^{7}$, confirm the analytics with much better accuracy, as the small-endpoint behavior follows the expected power law with exponent $\phi=1 / 2$ very well, see Fig. 4. Also for the case $H=3 / 4$, the behavior close to the origin matches the expected $P(y) \sim y^{\phi}$ behavior with $\phi=1 / 3$ very well, see Fig. 5 .

For the subdiffusive case the convergence with the length $L$ of the path is slower. We first consider the case $H=2 / 5$, see Fig. 6. For the longest walk, the behavior close to the origin follows a power law $P(y) \sim y^{\phi_{\text {eff }}}$, but the exponent $\phi_{\text {eff }}=1.44(1)$ (obtained from fitting a power law in the region $y \in[0.03,0.2])$ is slightly smaller than the predicted value $\phi=(1-H) / H=3 / 2$. A better estimation is obtained by performing a finite-length 


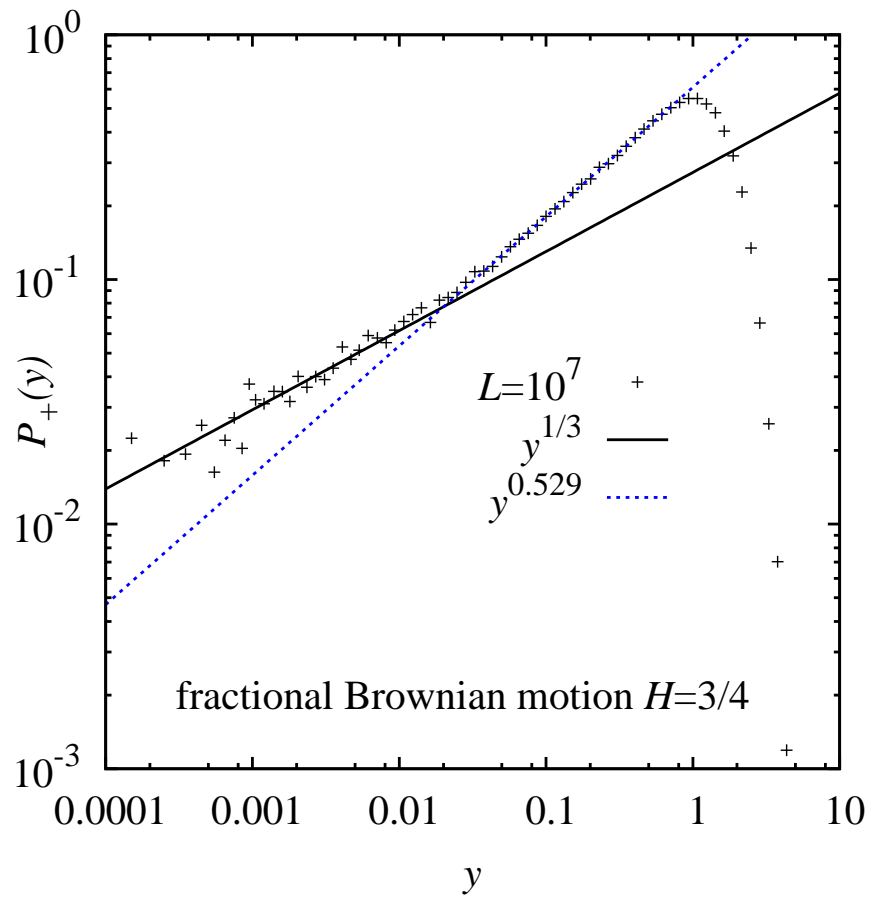

FIG. 5: (color online) Distribution $P_{+}(y)$ of rescaled endpoints $y=x / L^{H}$ for non-absorbed fBms (Hurst exponent $H=3 / 4)$.

extraplation of the form

$$
\phi_{\mathrm{eff}}(L)=\phi+c L^{-b}
$$

for the effective exponent as a function of the length $L$, see Fig. 7 When fitting Eq. (11) to the data, we obtained $\phi=1.50(4)$ (and $b=0.23(4)$ ), in prefect agreement with the prediction.

Finally, we turn to the most difficult case whith $H=$ $1 / 4$ where we expect that $P_{+}(y)$ vanishes as $y^{3}$ as $y \rightarrow 0$. Direct simulations on this process (for restricted sizes) are not conclusive and a scaling behavior $\sim y^{2}$ was found to be consistent with the data [26, 31]. Using our Markov chain approach we can see the finite size effects remain important even for long processes (see Fig. 8). Again, like in the case $H=2 / 5$, we observed power-law behvior close to the origin with an effective exponent $\phi_{\mathrm{eff}}(L)$. From $L=10^{5}$ on, the effective exponent is above 2 and is growing with walk length $L$. Hence, previous claim of a quadratic behavior can be discarded clearly. Here the extrapolation according to Eq. (11) yields $\phi=3.3(3)$ with a very slow convergence $b=0.08(2)$. Hence, even if one was able to study extremely long walks like $L=10^{11}$, one would observe $\phi_{\text {eff }} \approx 2.88$. Thus, the observed extrapolated exponent is also compatible within error bars with the predicted value $\phi=3$, but with lower accuracy due to stronger finite-length correction.

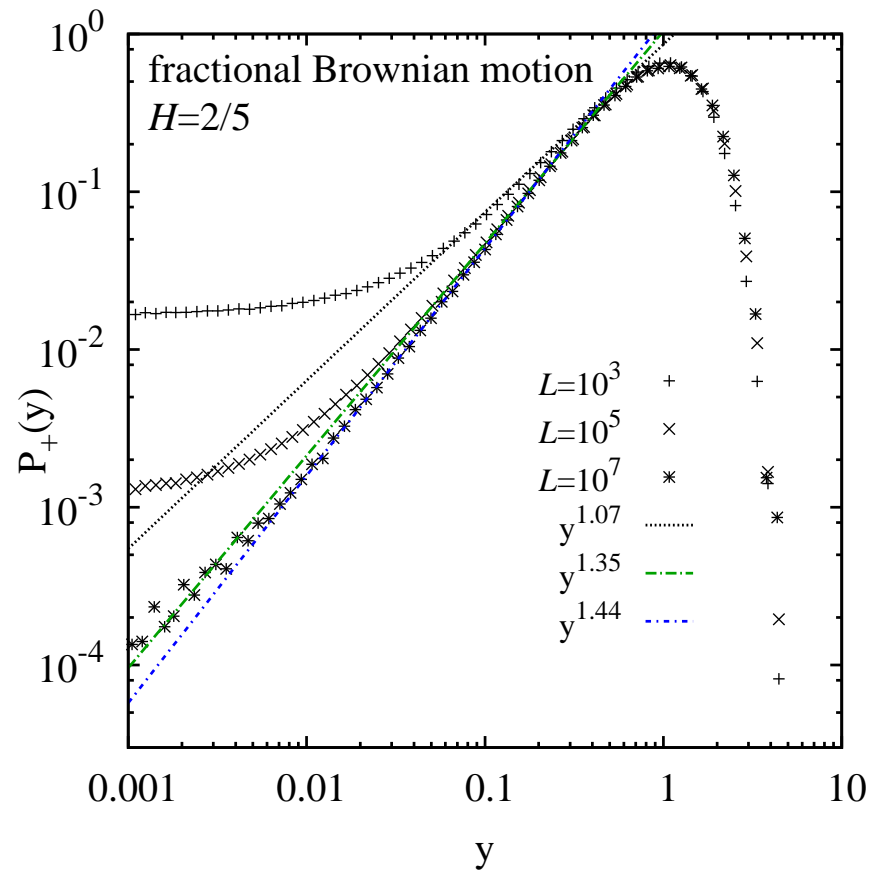

FIG. 6: (color online) Distribution $P_{+}(y)$ of rescaled endpoints $y=x / L^{H}$ for non-absorbed fBms (Hurst exponent $H=2 / 5)$

\section{SUMMARY}

We have introduced a Markov-chain Monte Carlo approach to study numerically fractional Brownian motion in the presence of an absorbing boundary via generating finite-step random walks with correlated disorder. Our approach allowed us to study long walks up to $L=10^{7}$ steps. For the test case $H=1 / 2$ the result for the distribution $P_{+}(y)$ of the rescaled endpoints $y$ of the walks agrees in the limit $L \rightarrow \infty$ with the exacty analytic result. We also derived analytical expression for the fite-length corrections, which turn also to be compatible with the numerical results, better with increasing step number $L$.

In the main part of our work, we studied fractional Brownian motion where we find for $y \rightarrow 0$ power-law behaviors $P_{+}(y) \sim y^{\phi}$. For the superdiffusive cases, $H=2 / 3$ and $H=3 / 4$, we observed for long walks $L=10^{7}$ that the measured exponents match the analytical prediction $\phi=(1-H) / H$ with very good accuracy.

For the subdiffusives cases $H=2 / 5$ and $H=1 / 4$ we found strong finite-length effects which can be described via an effective exponent $\phi_{\text {eff }}(L)$. Hence we could not observe the limiting exponent directly, but in both cases we found via a power-law extrapolation a convergence to the predicted values $\phi=(1-H) / H$. 


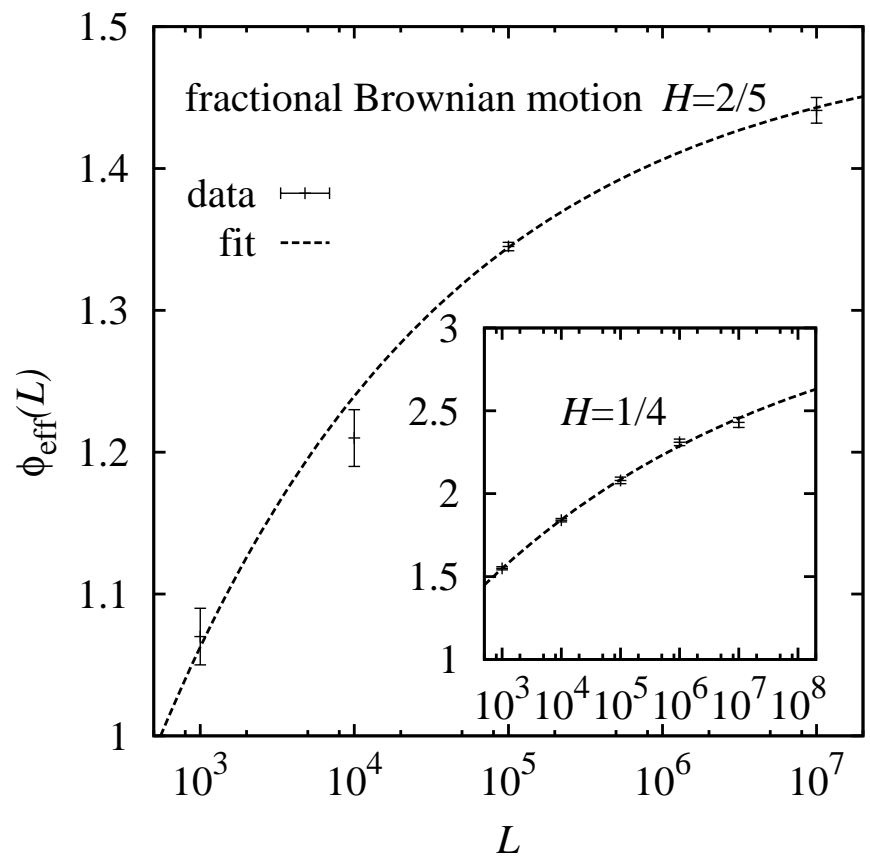

FIG. 7: Effective exponent $\phi_{\text {eff }}$ as a function of the walk length $L$ for $H=3 / 4$. The line shows a fit to the function $\phi_{\text {eff }}(L)=\phi+a L^{-b}$. Inset: for the case $H=1 / 4$.

\section{ACKNOWLEDGMENTS}

We thank Silivo Franz for interesting discussion about detailed balance of the approach, leading to the concise presentation given here. A. K. H. acknowledges the hospitality of the Aspen Center for Physics, which is supported by the National Science Foundation Grant No. PHY-1066293. The simulations were performed at the University of Oldenburg HERO high-performance computing facility which is funded by the DFG, INST 184/108-1 FUGG and the Ministry of Science and Culture (MWK) of the Lower Saxony state. A. K. H. thanks the Université Paris Sud and in particular Marc Mézard for the hospitality during several visits. S. N. M. would like to acknowledge support by ANR grant 2011-BS04013-02 WALKMAT. S. N. M and A. R. acknowledge support from the Indo-French Centre for the Promotion of Advanced Research under Project 4604-3.

\section{Appendix A: Derivation of Eq. 8 and Eq. 9}

We consider a random walk starting at the origin. Its position at discrete time steps evolves via

$$
x_{n}=x_{n-1}+\eta_{n}
$$

starting from $x_{0}=0$. The random variables $\eta_{n}$ 's are independent and identically distributed noises, each drawn from a symmetric and continuous probability density function (pdf) $f(\eta)$. Let $p_{L}(x)$ denote the probability

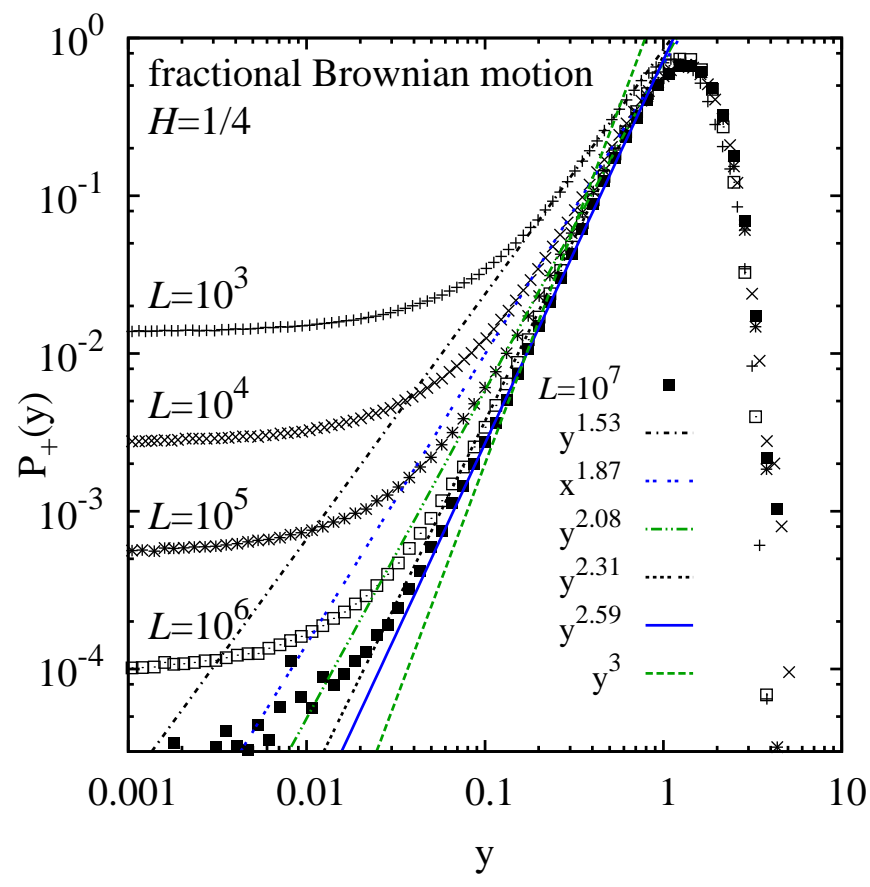

FIG. 8: (color online) Distribution $P_{+}(y)$ of rescaled endpoints $y=x / L^{H}$ for non-absorbed fBms (Hurst exponent $H=1 / 4)$

density that the particle arrives at $x$ at step $L$ while staying above 0 at all intermediate steps. An exact expression for $p_{L}(x)$, or rather for its generating function, is known explicitly for arbitrary jump density $f(\eta)$ and is given by [32]

$$
\int_{0}^{\infty} d x e^{-\lambda x} \sum_{L=0}^{\infty} p_{L}(x) s^{L}=\phi(s, \lambda)
$$

with

$$
\varphi(s, \lambda)=\exp \left(-\frac{\lambda}{\pi} \int_{0}^{\infty} \frac{\ln [1-s \hat{f}(k)]}{k^{2}+\lambda^{2}} d k\right)
$$

where $\hat{f}(k)=\int_{-\infty}^{\infty} e^{i k \eta} f(\eta) d \eta$ is the Fourier transform of the noise density. Our goal is to extract the leading (and subleading) scaling behavior of $p_{L}(x)$ for large $L$ from Eq. (A2).

To make progress, it is useful to consider an alternative expression for $\phi(s, \lambda)$ derived in Ref. [33], valid for all $f(\eta)$ 's with a finite variance $\sigma^{2}=\int_{\infty}^{\infty} \eta^{2} f(\eta) d \eta$,

$$
\begin{aligned}
& \phi(s, \lambda)=\frac{1}{[\sqrt{1-s}+\sigma \lambda \sqrt{s / 2}]} \times \\
& \times \exp \left[-\frac{\lambda}{\pi} \int_{0}^{\infty} \frac{d k}{\lambda^{2}+k^{2}} \ln \left(\frac{1-s \hat{f}(k)}{1-s+s \sigma^{2} k^{2} / 2}\right)\right] .
\end{aligned}
$$

We next consider the scaling limit when $x \rightarrow \infty, L \rightarrow \infty$, with the ratio $y=x / \sqrt{L}$ fixed. In the Laplace place, this 
corresponds to taking the limit $\lambda \rightarrow 0, s \rightarrow 1$, keeping the ratio $\lambda / \sqrt{1-s}$ fixed. Taking this scaling limit in Eq. (A4), one gets

$$
\phi(s, \lambda) \rightarrow \frac{1-c \lambda}{\sqrt{1-s}+\sigma \lambda / \sqrt{2}}
$$

where $c$ is a constant with the following expression 33 , 34

$$
c=\frac{1}{\pi} \int_{0}^{\infty} \frac{d k}{k^{2}} \ln \left[\frac{1-\hat{f}(k)}{\sigma^{2} k^{2} / 2}\right] .
$$

Substituting the scaling-limit expression of $\phi(s, \lambda)$ from Eq. (A5) on the right hand side of Eq. (A2) and inverting the Laplace transform with respect to $\lambda$ gives,

$$
\sum_{L=0}^{\infty} p_{L}(x) s^{L} \approx \frac{\sqrt{2}}{\sigma}\left[1+\frac{\sqrt{2}}{\sigma} c \sqrt{1-s}\right] e^{-\sqrt{2(1-s)} x / \sigma},
$$

valid in the scaling limit $s \rightarrow 1, x \rightarrow \infty$ but keeping the product $\sqrt{1-s} x$ fixed. Next, one can invert this generating function with respect to $s$, using Cauchy's inversion formula. Skipping details, we find that the two leading terms, in the scaling limit where $x \rightarrow \infty, L \rightarrow \infty$, but keeping $y=x / \sqrt{L}$ fixed, are given by

$$
p_{L}(x) \approx \frac{1}{\sigma^{2} \sqrt{\pi} L}\left[y e^{-y^{2} / 2 \sigma^{2}}-\frac{c}{\sqrt{L}} e^{-y^{2} / 2 \sigma^{2}}\right] .
$$

The conditional probability $P_{L}(x)$ (probability density to reach the position $x$ given that it has survived up to $L$ steps) is defined as

$$
P_{L}(x)=\frac{p_{L}(x)}{\int_{0}^{\infty} p_{L}(x) d x}
$$

Substituting the scaling behavior for $p_{L}(x)$ from Eq. (A8) in the above definition, we find that $P_{L}(x)$ has the following scaling behavior

$$
P_{L}(x) \rightarrow \frac{1}{\sqrt{L}} P_{+}(y, L)
$$

with $y=x / \sqrt{L}$ and

$$
P_{+}(y, L)=f_{0}(y)-\frac{c}{\sqrt{L}} f_{1}(y)+O(1 / L)
$$

where

$$
\begin{aligned}
& f_{0}(y)=\frac{y}{\sigma^{2}} e^{-y^{2} / 2 \sigma^{2}} \\
& f_{1}(y)=e^{-y^{2} / 2 \sigma^{2}}-\frac{2}{\pi \sigma} y e^{-y^{2} / 2 \sigma^{2}}
\end{aligned}
$$

and the constant $c$ is given by Eq. (A6). For the special case of the Gaussian jump density, $f(\eta)=e^{-\eta^{2} / 2} / \sqrt{2 \pi}$ (with $\sigma^{2}=1$ ), one can evaluate the constant $c$ in Eq. (A6) explicitly [33]

$$
c=\frac{\zeta(1 / 2)}{\sqrt{2 \pi}}=-0.582597 \ldots
$$

In this case, in particular, putting $y=0$ we get

$$
P_{+}(0, L) \approx-\frac{c}{\sqrt{L}}=\frac{0.582597 \ldots}{\sqrt{L}}
$$

which is consistent with our simulations.
[1] B. B. Mandelbrot and J. W. van Ness, SIAM Review 10, 422 (1968).

[2] Y. Kantor and M. Kardar, Phys. Rev. E 69, $021806 \quad$ (2004), URL http://link.aps.org/doi/10.1103/PhysRevE.69.021806

[3] J. Krug, H. Kallabis, S. N. Majumdar, S. J. Cornell, A. J. Bray, and C. Sire, Phys. Rev. E 56, 2702 (1997), URL http://link.aps.org/doi/10.1103/PhysRevE.56.2702

[4] T. E. Harris, Journal of Applied Probability 2, pp. 323 (1965), ISSN 00219002, URL http://www . jstor.org/stable/3212197

[5] S. N. Majumdar and M. Barma, Phys. Rev. B 44, $5306 \quad$ (1991), URL http://link.aps.org/doi/10.1103/PhysRevB.44.5306

[6] J.-C. Walter, A. Ferrantini, E. Carlon, and C. Vanderzande, Phys. Rev. E 85, 031120 (2012), URL http://link.aps.org/doi/10.1103/PhysRevE.85.031120

[7] A. Zoia, A. Rosso, and S. N. Majumdar, Phys. Rev. Lett. 102, 120602 (2009), URL http://link.aps.org/doi/10.1103/PhysRevLett.102.120602
[8] D. Panja and G. T. Barkema, The Journal of Chemical Physics 132, 014902 (pages 10) (2010), URL http://link. aip.org/link/?JCP/132/014902/1.

[9] D. Panja, G. T. Barkema, and R. C. Ball, Journal of Physics: Condensed Matter 19, 432202 (2007), URL http://stacks.iop.org/0953-8984/19/i=43/a=432202

[10] J. Szymanski and M. Weiss, Phys. Rev. Lett. 103, $038102 \quad$ (2009), URL http://link.aps.org/doi/10.1103/PhysRevLett.103.038102.

[11] S. C. Weber, A. J. Spakowitz, and J. A. Theriot, Phys. Rev. Lett. 104, 238102 (2010), URL http://link.aps.org/doi/10.1103/PhysRevLett.104.238102.

[12] J.-H. Jeon, H. M.-S. Monne, M. Javanainen, and R. Metzler, Phys. Rev. Lett. 109, 188103 (2012), URL http://link.aps.org/doi/10.1103/PhysRevLett.109.188103.

[13] L. Lizana, T. Ambjörnsson, A. Taloni, E. Barkai, and M. A. Lomholt, Phys. Rev. E 81, 051118 (2010), URL http://link.aps.org/doi/10.1103/PhysRevE.81.051118

[14] R. García-García, A. Rosso, and G. Schehr, $\begin{array}{lllll}\text { Phys. Rev. E 81, } 010102 & \text { (2010), URL }\end{array}$ 
http://link.aps.org/doi/10.1103/PhysRevE.81.010102

[15] G. Oshanin, A. Rosso, and G. Schehr (2012).

[16] R. Cakir, P. Grigolini, and A. A. Krokhin, Phys. Rev. E 74, 021108 (2006), URL http://link.aps.org/doi/10.1103/PhysRevE.74.021108

[17] I. Goychuk, Phys. Rev. E 80, 046125 (2009), URL http://link.aps.org/doi/10.1103/PhysRevE.80.046125

[18] J.-H. Jeon and R. Metzler, Phys. Rev. E 81, 021103 (2010), URL http://link.aps.org/doi/10.1103/PhysRevE.81.021103

[19] K. J. Wiese, S. N. Majumdar, and A. Rosso, Phys. Rev. E 83, 061141 (2011), URL http://link.aps.org/doi/10.1103/PhysRevE.83.061141

[20] A. T. A. Wood and G. Chan, Journal of Computational and Graphical Statistics 3, 409 (1994).

[21] C. R. Dietrich and G. N. Newsam, SIAM J. Sci. Comput. 18, 1088 (1997), ISSN 1064-8275, URL http://dx.doi.org/10.1137/S1064827592240555

[22] L. E. Wittig and A. K. Sinha, J. Acoust. Soc. Amer. 58, 630 (1975), URL http://link.aip.org/link/?JAS/58/630/1.

[23] M. Galassi, J. Davies, J. Theiler, B. Gough, G. Jungman, M. Booth, and F. Rossi, GNU Scientific Library Reference Manual (Network Theory Ltd., Bristol, UK, 2006), URL http: //www . gnu . org/software/gsl/

[24] S. N. Majumdar, Current Science 77, 370 (1999).

[25] A. J. Bray, S. N. Majumdar, and G. Schehr, Persistence and first-passage properties in non-equilibrium systems (2013), preprint arXiv:1304.1195.

[26] A. Amitai, Y. Kantor, and M. Kardar, Phys. Rev. E 81, 011107 (2010), URL
http://link.aps.org/doi/10.1103/PhysRevE.81.011107

[27] A. K. Hartmann, Phys. Rev. E 65, 056102 (2002).

[28] A. Engel, R. Monasson, and A. K. Hartmann, J. Stat. Phys. 117, 387 (2004).

[29] A. K. Hartmann, Eur. Phys. J. B 84, 627 (2011).

[30] A. K. Hartmann, Practical Guide to Computer Simulations (World Scientific, Singapore, 2009).

[31] Y. Kantor and M. Kardar, Phys. Rev. E 76, $061121 \quad$ (2007), URL http://link.aps.org/doi/10.1103/PhysRevE.76.061121.

[32] V. V. Ivanov, Astron. Astrophys. 286, 328 (1994).

[33] A. Comtet and S. N. Majumdar, Journal of Statistical Mechanics: Theory and Experiment 2005, P06013 (2005), URL http://stacks.iop.org/1742-5468/2005/i=06/a=P06013

[34] S. N. Majumdar, A. Comtet, and R. M. Ziff, Journal of Statistical Physics 122, 833 (2006), ISSN 0022-4715, URL http://dx.doi.org/10.1007/s10955-005-9002-x

[35] T. Dieker, URL http://www2.isye.gatech.edu/ adieker3/fbm/ind

[36] Better results for direct sampling can be obtained by making use of the fact for $\mathrm{fBm}$ the matrix $C$ is also a Toeplitz matrix. For Toeplitz matrices efficient numerical methods allow to avoid the full diagonalization of $C$. An example is given by the Levinson algorithm (for a practical implementation of Levinson's algorithm see [35].). However here we use a Markov Chain sampling (not compatible with the Levinson algorithm) which is more efficient in presence of absorption. 